\title{
Associação entre eficiência alimentar e ganho em acabamento de carcaça de bovinos da raça Nelore
}

[Association between feed efficiency and carcass finishinging gain of Nellore cattle]

\author{
M.M.A. Gomes ${ }^{1}$, C.U. Faria ${ }^{2}$, B.S.B.R. Bezerra ${ }^{3}$, J.M. Miguel ${ }^{4,3}$, \\ G.C. Borges ${ }^{3}$, E.D.M. Mendes ${ }^{4}$
}
'Aluna de pós-graduação - Universidade Federal de Uberlândia - Uberlândia, MG
${ }^{2}$ Universidade Federal de Uberlândia - Uberlândia, MG
${ }^{3}$ Programa de graduação - Zootecnia - Universidade Federal de
Uberlândia - Uberlândia, MG
${ }^{4}$ Embrapa Pecuária Sudeste - São Carlos, SP

\section{RESUMO}

Objetivou-se estimar a associação fenotípica entre a eficiência alimentar e o ganho em acabamento de carcaça de touros jovens da raça Nelore. Utilizaram-se informações de 98 touros jovens da raça Nelore, participantes de duas provas de eficiência alimentar que empregaram o sistema de cochos eletrônicos Growsafe $^{\circledR}$, as quais foram realizadas nos anos de 2015 e 2016, na fazenda Capim Branco, da Universidade Federal de Uberlândia. As provas de eficiência alimentar tiveram duração de 91 dias, sendo 21 dias de adaptação e 70 dias de avaliação. Os animais apresentavam idade média ao início e ao término do confinamento de 19,98 e 22,16 meses e peso inicial e final médio de 428,17 e 542,27kg, respectivamente. Verificou-se que os animais mais eficientes depositaram menos gordura, e os animais menos eficientes depositaram mais gordura durante o período de avaliação em confinamento. Observouse que há uma associação fenotípica de baixa magnitude $(0,29)$ entre o consumo alimentar residual (CAR) e o ganho em acabamento de carcaça (GACAB). Assim, pode-se inferir que, ao serem selecionados menores valores de CAR, haverá alguma interferência negativa em GACAB. Concluiu-se que animais mais eficientes (CAR negativo) podem apresentar menor deposição de gordura subcutânea, influenciando na qualidade de carcaça de bovinos da raça Nelore.

Palavras-chave: consumo alimentar residual, gordura subcutânea, bovinos de corte

\begin{abstract}
This study aimed to estimate the phenotypic association between feed efficiency and gain in carcass finishing of young Nelore bulls. Information from 98 young Nelore bulls participating in two feed efficiency tests using the Growsafe ${ }^{\circledR}$ electronic trough system, carried out in the years 2015 and 2016, at the Capim Branco farm of the Universidade Federal de Uberlandia was used.The feed efficiency tests lasted for 91 days, with 21 days of adaptation and 70 days of evaluation. The animals had a mean age at the beginning and end of the confinement of 19.98 and 22.16 months, and mean weight initial and end of 428.17 and $542.27 \mathrm{~kg}$, respectively. It was verified that most efficient animals deposited less fat, and the efficient animals deposited more fat during the evaluation period at the confinement. It was observed that there is a low magnitude phenotypic association (0.29) between the residual feed intake (RFI) and the gain in carcass finishing (GACAB). Thus, it can be inferred that by selecting lower values of residual feed intake there will be some negative interference in GACAB. It was concluded that the most efficient animals (negative RFI) may present lower deposition of subcutaneous fat, influencing the carcass quality of Nelore cattle.
\end{abstract}

Keywords: residual feed intake, subcutaneous fat, beef cattle

Recebido em 24 de janeiro de 2019

Aceito em 13 de maio de 2019

E-mail: mmundim95@yahoo.com.br 


\section{INTRODUÇÃO}

A agropecuária exerce funções essenciais no avanço da economia de um país, sendo elas desde o fornecimento de alimentos para a população até a geração de empregos, renda e mercado consumidor para bens industrializados (Boas..., 2007). Entre as atividades de destaque do agronegócio nacional, a bovinocultura de corte tem se sobressaído na economia brasileira e, ainda, assumindo posição de liderança no mercado mundial de exportação de carnes (Anuário..., 2017).

Apesar dos avanços na bovinocultura de corte, é imprescindível aumentar os índices de produtividade e rentabilidade do setor, equilibrando genótipo, ambiente de criação, mercado e redução de custos para se manter competitivo e economicamente atraente no mercado (Almeida, 2005). A alimentação dos animais representa o fator de maior dispêndio, o que corresponde de 70 a $90 \%$ dos custos operacionais totais, dentro do sistema de produção (Corvino, 2010). Portanto, faz-se necessário buscar estratégias de redução no consumo de alimentos na produção animal, que impactará positivamente tanto em fatores econômicos quanto em fatores ambientais. No entanto, é preciso associar mecanismos que aumentem a eficiência alimentar e que não prejudiquem características de desempenho, reprodução e qualidade de carcaça (Almeida, 2005). A eficiência alimentar em bovinos de corte é conceituada como a capacidade que o animal tem de converter o alimento ingerido em produto de origem animal, sendo em carne ou carcaça (Gomes et al., 2012).

Um dos parâmetros indicadores de eficiência alimentar é o consumo alimentar residual (CAR), que foi proposto, primeiramente, por Koch et al. (1963). O CAR é calculado pela diferença entre a ingestão de matéria seca (IMS) observada e a IMS estimada por equação de regressão múltipla baseada no peso vivo médio metabólico e no ganho médio diário (GMD) dos animais do mesmo grupo contemporâneo (Arthur et al., 2001). Assim, animais que apresentam CAR negativo são os que apresentam um consumo menor que o previsto, sendo, portanto, mais eficientes; ao contrário disso, animais de CAR positivo são aqueles que ingerem maior quantidade de matéria seca do que o esperado.
A mensuração do CAR é vantajosa e rentável para todos os sistemas de produção de carne bovina e pode ser incluída em programas de seleção, pois as estimativas de herdabilidade variam de moderada a alta (Moore et al., 2009; Mcgee et al., 2013; Gomes et al., 2012). Dessa forma, a seleção para o CAR poderá levar à produção de animais com menor consumo de alimentos, mas, de acordo com Basarab et al. (2003), como consequência, poderá acarretar a seleção de animais com menor teor de gordura subcutânea. Segundo Santana et al. (2014), essa redução da gordura subcutânea impacta, negativamente, na qualidade da carcaça, bem como no sabor e na suculência da carne bovina. Entretanto, são escassas as informações científicas sobre as associações entre a eficiência alimentar e o acabamento de carcaça em bovinos de corte, especialmente, na raça Nelore. Diante disso, o objetivo deste estudo foi estimar a associação fenotípica entre a eficiência alimentar e o ganho em acabamento de carcaça de touros jovens da raça Nelore participantes de provas de eficiência alimentar.

\section{MATERIAL E MÉTODOS}

Para a realização do presente estudo, foram consideradas as informações de 98 touros jovens da raça Nelore, puros de origem (PO), participantes de duas provas de eficiência alimentar utilizando o sistema de cochos eletrônicos Growsafe ${ }^{\circledR}$, realizadas nos anos de 2015 e 2016, na fazenda Capim Branco, da Universidade Federal de Uberlândia, em Uberlândia, MG. Foram avaliadas as características de consumo alimentar residual (CAR), acabamento de carcaça mensurada ao início $\left(\mathrm{ACAB}_{\mathrm{I}}\right)$ e término $\left(\mathrm{ACAB}_{\mathrm{F}}\right)$ do confinamento, ganho em acabamento de carcaça (GACAB), peso ao final do confinamento $\left(\mathrm{PESO}_{\mathrm{F}}\right)$, ganho médio diário $(\mathrm{GMD})$ e ingestão de matéria seca (IMS). As medidas de ACAB foram avaliadas por ultrassonografia, no início e no término do experimento, sendo o GACAB calculado como a diferença dessas medidas de ACAB durante o período de confinamento.

O confinamento era estruturado em uma área de $1.680 \mathrm{~m}^{2}(42 \mathrm{~m} \times 40 \mathrm{~m})$, dividida em dois curraletes, com um bebedouro central de 2.600 litros. Cada curralete continha quatro cochos eletrônicos (com cobertura) do sistema Growsafe $^{\circledR}$. As provas de eficiência alimentar 
tiveram duração de 91 dias, sendo os primeiros 21 dias para adaptação dos animais à dieta e ao sistema de confinamento e os demais 70 dias para mensuração do consumo. Os animais participantes das provas representavam um grupo de contemporâneos, com idade média ao início e término da avaliação de 19,98 e 22,16 meses, respectivamente, e peso inicial e final médio de 428,17 e $542,27 \mathrm{~kg}$, respectivamente.

Os animais foram pesados no início e término de cada prova e, também, em intervalos de 14 dias, sem a realização de jejum. A dieta foi fornecida ad libidum, em forma de uma única ração total, visando a ganhos médios diários de $1,2 \mathrm{~kg} /$ animal $/$ dia. A formulação da ração foi de $60 \%$ de volumoso e $40 \%$ de concentrado, atendendo as recomendações do NRC (Nutrient..., 2016) para os ganhos esperados. A ração fornecida era de $60 \%$ de silagem de milho, $28 \%$ de milho moído, $8 \%$ de farelo de soja, $1 \%$ de ureia e $3 \%$ de núcleo mineral, a qual fornecia aproximadamente $13 \%$ de proteína bruta, $70 \%$ de NDT, $38 \%$ de CNF e 2,9\% de EE. O manejo de arraçoamento era realizado duas vezes ao dia; diariamente se acompanhavam as sobras dos cochos e eram feitos ajustes, caso necessário, a fim de garantir sobras em torno de 5\% do fornecido. Em intervalos de 14 dias, foram realizadas análises bromatológicas de MS (matéria seca).

Para a avaliação de eficiência alimentar, utilizouse a informação de consumo alimentar residual (CAR). Conforme descrito por Gomes et al. (2012), o CAR é calculado como a diferença de ingestão de matéria seca $\left(\mathrm{IMS}_{\mathrm{obs}}\right)$ observada durante a prova zootécnica e a ingestão de matéria seca esperada $\left(\mathrm{IMS}_{\mathrm{esp}}\right)$ baseada no peso corporal e no ganho em peso. O peso vivo do animal é definido como peso vivo médio metabólico (PVMM) e foi calculado conforme descrito a seguir:

$$
P V M M=\left(\frac{P V I+P V F}{2}\right)^{0,75},
$$

em que: PVI é o peso vivo inicial e PVF é o peso vivo final à prova de avaliação para eficiência alimentar. As informações de ingestão de matéria seca $\left(\mathrm{IMS}_{\mathrm{obs}}\right)$, ganho médio diário (GMD) e peso vivo metabólico (PVMM) de todos os animais em teste foram utilizadas para predição dos coeficientes ( $\beta$ ) da equação de regressão linear múltipla, conforme apresentada:

$$
I M S=\beta_{0}+\left(\beta_{1} \times G M D\right)+\left(\beta_{2} \times P V M M\right)
$$

Estabelecida a equação, calculou-se a ingestão de matéria seca esperada ( IMS $_{\text {esp }}$ ) para cada animal. Em seguida, o cálculo do CAR (em $\mathrm{kg}$ ) foi dado por:

$$
C A R=I M S_{o b s}-I M S_{e s p}
$$

Ao ajustar o CAR para peso vivo metabólico (PVMM), ele se torna independente dessa característica, em que, se forem selecionados animais mais eficientes para CAR, não haverá interferência no crescimento e no tamanho adulto do rebanho (Chaves, 2013). Para a mensuração do acabamento de carcaça, foram coletadas imagens de ultrassonografia por meio de um aparelho ALOKA $500 \mathrm{v}$, com sonda linear de $17,2 \mathrm{~cm}$, de $3,5 \mathrm{MHz}$ e um acoplador acústico em conjunto com um sistema de captura de imagens (Blackbox, Bionitrics, Inc., Ames, IA, EUA). As coletas de imagens foram feitas ao início e ao final do confinamento, no qual a espessura de gordura subcutânea no dorso (EG) foi medida no músculo Longissimus dorsi, entre a $12^{\mathrm{a}}$ e $13^{\mathrm{a}}$ costelas, e a espessura de gordura subcutânea na garupa (EGP8) foi medida na garupa entre os músculos localizados entre o ílio e o ísquio. Essas imagens foram coletadas e interpretadas pelo laboratório responsável pela garantia da qualidade dos dados (Aval Serviços Tecnológicos $\mathrm{S} / \mathrm{S}$ ). A característica ACAB é um índice que contempla $65 \%$ da EGP8 e $35 \%$ da EG.

Para a realização das análises descritivas e estatísticas, utilizou-se o programa Statistical Analysis System (SAS, 2004). Também foi utilizado o pacote Microsoft Excel para preparação do arquivo de dados e confecção de gráficos. Para a estimação dos coeficientes de correlação de Pearson entre o consumo alimentar residual e o ganho em acabamento de carcaça, foi usado o programa Statistical Analysis System (SAS, 2004). A análise descritiva das características avaliadas é apresentada na Tab. 1. 
Tabela 1. Análise descritiva das características de idade ao início (IDADE $)_{\mathrm{I}}$ e ao final (IDADE (ID $_{\mathrm{F}}$ do confinamento, peso inicial $\left(\mathrm{PESO}_{\mathrm{I}}\right)$, peso final $\left(\mathrm{PESO}_{\mathrm{F}}\right)$, acabamento de carcaça mensurado no início $\left(\mathrm{ACAB}_{\mathrm{I}}\right)$ e no final $\left(\mathrm{ACAB}_{\mathrm{F}}\right)$ do confinamento, ganho em acabamento nesse período (GACAB), ganho médio diário (GMD), ingestão de matéria seca (IMS) e consumo alimentar residual (CAR) em touros jovens da raça Nelore

\begin{tabular}{lccccc}
\hline Variável & Média & DP & CV $(\%)$ & Mínimo & Máximo \\
\hline IDADE $_{\mathrm{I}}$ (meses) & 19,98 & 0,58 & 2,94 & 18,76 & 20,93 \\
IDADE $_{\mathrm{F}}(\mathrm{meses})$ & 22,16 & 0,58 & 2,64 & 21,00 & 23,06 \\
PESO $_{\mathrm{I}}(\mathrm{kg})$ & 428,17 & 43,29 & 10,11 & 330,00 & 525,00 \\
PESO $_{\mathrm{F}}(\mathrm{kg})$ & 542,27 & 53,76 & 9,91 & 425,00 & 672,00 \\
$\mathrm{ACAB}_{\mathrm{I}}(\mathrm{mm})$ & 2,39 & 0,66 & 27,61 & 1,36 & 4,23 \\
$\mathrm{ACAB}_{\mathrm{F}}(\mathrm{mm})$ & 6,41 & 1,43 & 22,31 & 3,50 & 10,95 \\
$\mathrm{GACAB}(\mathrm{mm})$ & 4,02 & 1,18 & 29,35 & 1,68 & 7,71 \\
GMD $(\mathrm{kg} / \mathrm{dia})$ & 1,74 & 0,26 & 14,94 & 1,03 & 2,28 \\
IMS (kg de MS/dia) & 10,77 & 1,49 & 13,83 & 6,52 & 14,01 \\
CAR (kg de MS/dia) & $-0,01$ & 1,03 & NA & $-3,11$ & 2,04 \\
\hline
\end{tabular}

DP: desvio-padrão; CV: coeficiente de variação; NA: não avaliado.

Também foram elaboradas as classes de CAR, considerando-se 33 animais com CAR baixo $(33,7 \%), 32$ animais com CAR médio $(32,6 \%)$ e 33 animais com CAR alto $(33,7 \%)$. Com o intuito de verificar a influência das classes do consumo alimentar residual (baixo CAR, médio CAR e alto CAR) sobre o ganho em acabamento de carcaça (GACAB), foram realizadas análises de variância (ANOVA) utilizando-se o método dos quadrados mínimos, por meio do procedimento GLM (General Linear Model), em que o modelo estatístico é apresentado a seguir:

$$
y_{i j}=\mu+C A R_{i}+e_{i j},
$$

em que: $y_{i j}=$ valor observado (ganho em acabamento de carcaça); $\mu=$ média geral da característica fenotípica; $C A R=$ efeito do tratamento (classes de CAR); e $e^{e_{i j}}=$ vetor de efeitos residuais. A comparação das médias foi realizada pelo teste de Tukey, sendo considerada diferença estatística quando $\mathrm{P} \leq 0,05$ (Zar, 1999). $\mathrm{O}$ mesmo procedimento ou teste estatístico foi realizado com a ingestão de matéria seca (IMS), gerando classes de IMS alto, médio e baixo.

\section{RESULTADOS E DISCUSSÕES}

As médias fenotípicas do ganho em acabamento de carcaça (GACAB) nas classes (baixo, médio e alto) de CAR e IMS são apresentadas na Tab. 2.
Observou-se que as classes de CAR (baixo, médio e alto) influenciaram na deposição de gordura subcutânea dos animais em confinamento, ou seja, os animais mais eficientes (baixo CAR) depositaram menos gordura, e os animais menos eficientes (alto CAR) depositaram mais gordura durante o período de avaliação em confinamento. Esses resultados corroboram os encontrados por Basarab et al. (2003), os quais verificaram que animais ineficientes possuem maior deposição de gordura na carcaça, tendo ocorrido propensão à menor deposição de gordura em animais de CAR negativo. Entretanto, não houve diferença estatística em relação às classes de IMS com o GACAB (Tab. 2), portanto não se verificou relação entre o consumo e a deposição de gordura subcutânea durante o confinamento.

Tabela 2. Médias fenotípicas do ganho em acabamento de carcaça obtido em mm (GACAB) em relação às classes de consumo alimentar residual (CAR) e ingestão de matéria seca em kg (IMS) de touros jovens da raça Nelore

\begin{tabular}{ccc}
\hline Classe & $\begin{array}{c}\text { Nome da } \\
\text { classe }\end{array}$ & GACAB \\
\hline 1 & Baixo CAR & $3,55^{\mathrm{b}}$ \\
2 & Médio CAR & $4,07^{\mathrm{ab}}$ \\
3 & Alto CAR & $4,43^{\mathrm{a}}$ \\
1 & Baixo IMS & 3,71 \\
2 & Médio IMS & 3,95 \\
3 & Alto IMS & 4,38 \\
\hline
\end{tabular}

$\mathrm{Na}$ Tab. 3, foram apresentadas as associações fenotípicas entre as características de consumo 
alimentar residual (CAR), o acabamento de carcaça avaliado ao início e ao final do confinamento $\left(\mathrm{ACAB}_{\mathrm{I}}\right.$ e $\left.\mathrm{ACAB}_{\mathrm{F}}\right)$, o ganho em acabamento de carcaça no período de avaliação (GACAB), além das medidas de peso vivo final $\left(\mathrm{PESO}_{\mathrm{F}}\right)$, ganho médio diário (GMD) e ingestão de matéria seca (IMS). Observou-se que há uma associação fenotípica de baixa magnitude entre o CAR e o GACAB, resultante da diferença de ganho mensurada no início e no término do experimento (regime de confinamento). Assim, pode-se inferir que, ao se selecionarem menores valores de CAR, haverá alguma interferência negativa em ganho em acabamento, o que corrobora os estudos de Basarab et al. (2003), Farjalla (2009), Nascimento (2011) e Santana et al. (2014).

Tabela 3. Coeficiente de correlação de Pearson (acima da diagonal) entre as medidas de acabamento de carcaça (ACABI, ACABF), o ganho total em acabamento de carcaça (GACAB), o peso final (PESOF), o ganho médio diário, a ingestão de matéria seca (IMS) e o consumo alimentar residual (CAR) para touros jovens da raça Nelore. Abaixo da diagonal, estão representadas as significâncias das correlações

\begin{tabular}{lllllllll} 
& $\mathrm{N}$ & $\mathrm{ACAB}_{\mathrm{I}}$ & $\mathrm{ACAB}_{\mathrm{F}}$ & GACAB & PESO $_{\mathrm{F}}$ & GMD & IMS & CAR \\
\hline ACAB $_{\mathrm{I}}$ & 98 & 1 & 0,57 & 0,13 & 0,42 & 0,04 & $-0,07$ & $-0,12$ \\
$\mathrm{ACAB}_{\mathrm{F}}$ & 98 & $<0,0001$ & 1 & 0,89 & 0,10 & 0,17 & 0,17 & 0,18 \\
$\mathrm{GACAB}$ & 98 & 0,21 & $<0,0001$ & 1 & 0,09 & 0,18 & 0,24 & 0,29 \\
PESO $_{\mathrm{F}}$ & 98 & 0,68 & 0,34 & 0,36 & 1 & 0,65 & 0,66 & $-0,003$ \\
$\mathrm{GMD}$ & 98 & 0,67 & 0,10 & 0,08 & $<0,0001$ & 1 & 0,51 & 0,002 \\
$\mathrm{IMS}$ & 98 & 0,46 & 0,10 & 0,01 & $<0,0001$ & $<0,0001$ & 1 & 0,69 \\
$\mathrm{CAR}$ & 98 & 0,23 & 0,07 & 0,004 & 0,1 & 0,1 & $<0,0001$ & 1 \\
\hline
\end{tabular}

Verificou-se também (Tab. 3) que, praticamente, não há associação fenotípica entre o CAR e as características de acabamento de carcaça mensuradas no início do confinamento $\left(\mathrm{ACAB}_{\mathrm{I}}\right)$, o peso $\left(\mathrm{PESO}_{\mathrm{F}}\right)$ e o ganho médio diário (GMD) dos animais avaliados. Todavia, como esperado, o CAR é altamente associado com a ingestão de matéria seca (IMS). Estudos apontam que os animais mais eficientes para CAR, além de consumirem menos alimento do que o esperado em virtude de sua produção, apresentam os mesmos índices de desempenho, são menos prejudicados em condições estressantes e ainda detêm menores gastos com energia de mantença; apesar disso, podem apresentar menor deposição de gordura na carcaça quando comparados com os animais menos eficientes.

Os resultados encontrados neste estudo (Tab. 3) corroboram os de Nkrumah et al. (2004) e Basarab et al. (2003), os quais relataram que, na avaliação de eficiência produtiva, os animais com maior porcentagem de tecido magro são considerados mais eficientes quando comparados com animais com maior proporção de gordura. Além disso, evidenciaram correlações positivas entre CAR e deposição de gordura na carcaça e correlações negativas entre CAR e deposição de tecido muscular na carcaça, ou seja, animais ineficientes depositaram mais tecido adiposo na carcaça, assim como animais eficientes depositaram maior tecido muscular. No entanto, essa propensão de animais CAR negativo depositarem menos gordura na carcaça tem se tornado preocupante para estudiosos, visto que a seleção para animais mais eficientes pode gerar uma desvantagem na qualidade de carcaça.

McDonagh et al. (2001) propõem que o consumo predito seja calculado também em função de gordura subcutânea como forma de rever esse ajuste, pois pode ser uma influência negativa da qualidade de carcaça. Basarab et al. (2003) complementaram que a inclusão dessa medida resultará em correlação dessa medida com o CAR perto de zero. Desse modo, a seleção fundamentada no CAR não conduziria uma correlação com a deposição de gordura subcutânea na carcaça. No entanto, Corvino (2010) discorda da proposta, uma vez que ele considera que não há interferência do CAR nas características de carcaça.

Bridi (2010) acrescenta que, à medida que o animal cresce, a sua eficiência alimentar decresce. Primeiramente, porque os requerimentos nutricionais de mantença aumentam, já que é uma característica associada ao peso corporal, e, em segundo lugar, porque o tecido adiposo é 2,5 vezes mais caro, nutricionalmente, para ser depositado, visto que o tecido adiposo possui somente $10 \%$ de água, 
enquanto o tecido muscular possui $78 \%$ de água na sua composição. A eficiência de produção de proteína é $26,71 \%$ e de gordura é de $75,43 \%$, e aproximadamente $60 \%$ da energia consumida pode ser retida como gordura e $35 \%$ da energia consumida pode ser retida como proteína. Portanto, em peso de tecido depositado, o músculo exige menos energia, já que, para cada quatro gramas de tecido muscular depositado, três gramas são de água.

Nesse contexto, Basarab et al. (2003) relataram que animais mais eficientes (CAR negativo) apresentaram menor consumo de energia metabolizável e menor retenção de energia, produziram menos calor e apresentaram vísceras menores (fígado, abomaso e intestinos) quando comparados com os menos eficientes (CAR positivo). Segundo estudos de Santana et al. (2012), a provável menor deposição de gordura na carcaça de animais com CAR negativo insinua que a seleção para melhorar o CAR resultaria em animais com menores reservas corporais. Considerando-se matrizes, essas reservas são importantes para a manutenção, ainda mais quando criadas em regiões tropicais que são submetidas à sazonalidade de qualidade e disponibilidade de forragem. Dessa maneira, essa categoria poderia ser afetada negativamente e resultar em menores taxas reprodutivas do rebanho. Robinson e Oddy (2004) e Herd et al. (2004) asseguram que animais mais eficientes propendem a apresentar carcaças mais magras, com menor acabamento e com menor gordura intramuscular, além de apontar menor porcentagem de gordura abdominal.

A dispersão das informações de ganho em acabamento de carcaça (GACAB) em relação ao consumo alimentar residual (CAR) é apresentada na Fig. 1. Verificou-se uma linha de tendência crescente, ou seja, o aumento do GACAB pode proporcionar um aumento do CAR. Vale ressaltar que, no processo de seleção, objetiva-se identificar os animais do quadrante I, ou seja, animais geneticamente superiores para acabamento de carcaça (maior potencial de deposição de gordura subcutânea) e eficientes (menor consumo alimentar residual).

\section{CAR X GACAB}

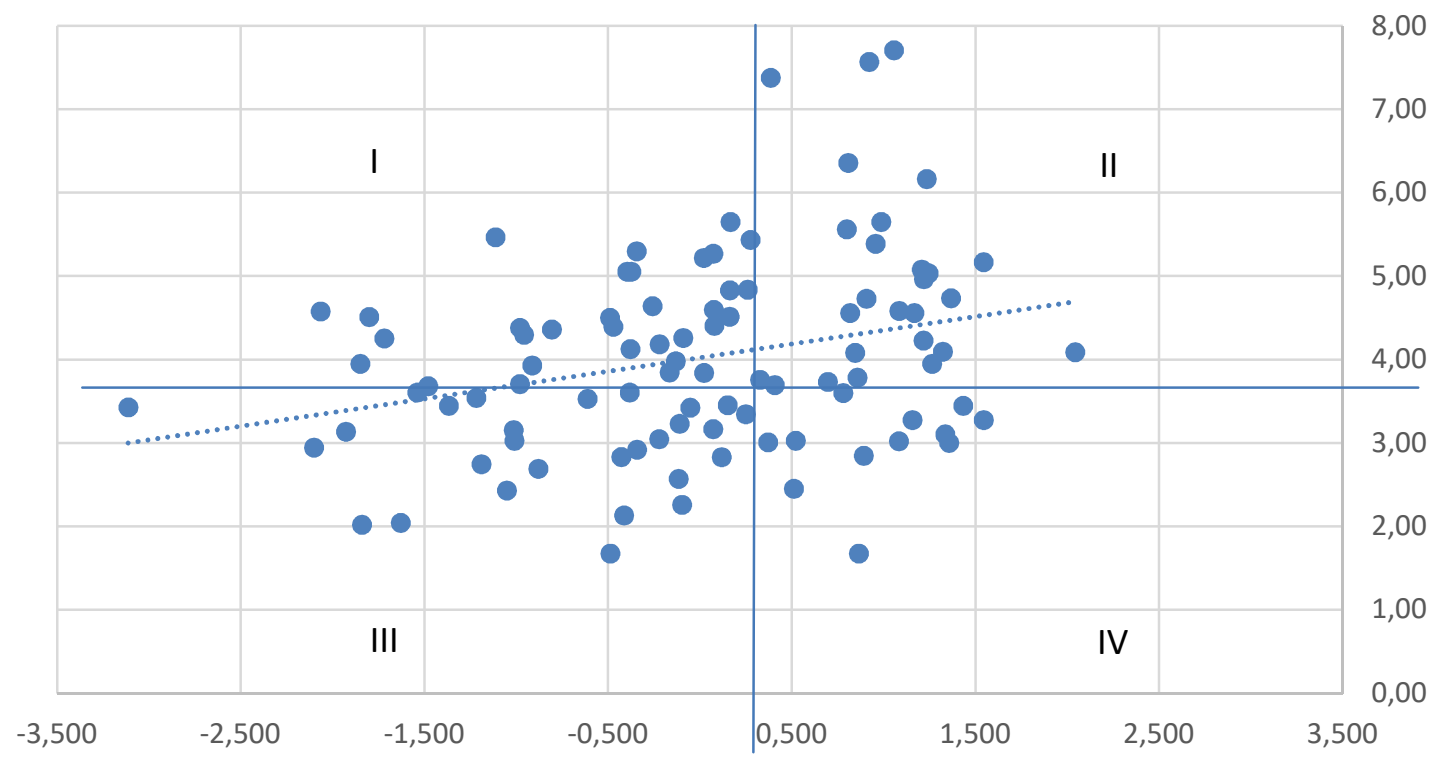

Figura 1. Avaliação da dispersão dos dados considerando-se o ganho em acabamento de carcaça (GACAB) e o consumo alimentar residual (CAR) de touros jovens da raça Nelore.

Recomenda-se a realização de novos estudos para comprovar se a seleção para o consumo alimentar resisual (CAR) não acarreta prejuízos para o ganho em acabamento de carcaça (ACAB) em bovinos da raça Nelore. 


\section{CONCLUSÃO}

Conclui-se que o ganho em acabamento de carcaça (GACAB) tem baixa associação positiva com o consumo alimentar resisual (CAR), indicando que animais mais eficientes (CAR negativo) podem apresentar menor deposição de gordura subcutânea, influenciando negativamente na qualidade de carcaça de bovinos da raça Nelore. No entanto, no processo de seleção, objetiva-se identificar os animais geneticamente superiores para acabamento de carcaça (maior potencial de deposição de gordura subcutânea) e eficientes (menor consumo alimentar residual).

\section{REFERÊNCIAS}

ALMEIDA, R. Consumo e eficiência alimentar de bovinos em crescimento. 2005. 181f. Tese (Doutorado em Ciência Animal e Pastagens) Escola Superior de Agricultura Luiz de Queiroz, Universidade de São Paulo, Piracicaba, SP.

ANUÁRIO da pecuária brasileira. São Paulo: Instituto FNP, 2017.

ARTHUR, P.F.; ARCHER, J.A.; JOHNSTON, D.J. et al. Genetic and phenotypic variance and covariance components for feed intake, feed efficiency, and other post weaning traits in Angus cattle. J. Anim. Sci., v.79, p.2805-2811, 2001.

BASARAB, J.A.; PRICE, M.A.; ALHUS, J.L. et al. Residual feed intake and body composition in young growing cattle. Can. J. Anim. Sci., v.83, p.189-204, 2003.

BOAS práticas agropecuárias de bovino de corte: sistemas de produção competitivos e acesso a mercados. Brasília: Embrapa Gado de Corte, $2007 . \quad$ Disponível em: $<$ http://www.agricultura.gov.br/arq_editor/7.pdf >. Acessado em: 03 mai. 2018.

BRIDI, A.M. Crescimento e desenvolvimento do tecido muscular. 2010. 14p. Disponível em: http://www.uel.br/pessoal/ambridi/Carnesecarcac asarquivos/Crescimentoedesenvolvimentomuscul ar.pdf. Acesso em: 12 ago. 2019
CHAVES, A.S. Relações entre eficiência alimentar e característica de carcaça, qualidade de carne, batimentos cardíacos e consumo de oxigênio em bovinos. 2013. 132f. Tese (Doutorado em Ciência animal e pastagens) Escola Superior de Agricultura Luiz de Queiroz, Universidade de São Paulo, Piracicaba, SP.

CORVINO, T.L.S. Caracterização do consumo alimentar residual e relações com desempenho e características de carcaça de bovinos nelore. 2010. 92f. Dissertação (Mestrado em Zootecnia) - Universidade Estadual Paulista, Botucatu, SP.

FARJALLA, Y.B. Desempenho, características de carcaça e qualidade de carne de bovinos Nelore estratificados pela eficiência através do consumo alimentar residual. 2009. 58f. Dissertação (Mestrado em Ciência animal e pastagens) - Escola Superior de Agricultura Luiz de Queiroz, Universidade de São Paulo, Piracicaba, SP.

GOMES, R.C.; SANTANA, M.H.A.; FERRAZ, J.B.S. et al. Ingestão de alimentos e eficiência alimentar de bovinos e ovinos de corte. Ribeirão Preto - SP: FUNPEC-RP, 2012. 81p.

HERD, R.M.; ODDY, V.W.; RICHARDSON, E.C. Biological basis for variation in residual feed intake in beef cattle.1. Review of potential mechanisms. Aust. J. Exp. Agric., v.44, p.423430, 2004.

KOCH, R.M.; SWIGER, L.A.; CHAMBERS, D. et al. Efficiency of feed use in beef cattle. $J$. Anim. Sci., v.22, p.486-494, 1963.

MCDONAGH, M.B.; HERD, R.M.; RICHARDSON, E.C. et al. Meat quality and the calpain system of feedlot steers following a single generation of divergent selection for residual feed intake. Aust. J. Exp. Agric., v.41, p.103-121, 2001.

MCGEE, M.; WELCH, C.M.; HALL, J.B. et al. Evaluation of Wagyu for residual feed intake: optimizing feed efficiency, growth, and marbling in Wagyu cattle. Prof. Anim. Sci., v.29 ,p.51-56, 2013.

MOORE, S.S.; MUJIBI, F.D.; SHERMAN, E.L. Molecular basis for residual feed intake in beef cattle. J. Anim. Sci., v.87, p.41-47, 2009. 
NASCIMENTO, M.L. Eficiência alimentar $e$ suas associações com lucro, características de carcaça e qualidade de carne de bovinos Nelore. 2011. 119f. Tese (Doutorado em Ciência animal e pastagens) - Escola Superior de Agricultura Luiz de Queiroz, Universidade de São Paulo, Piracicaba, SP.

NKRUMAH, J.D.; BASARAB, J.A.; PRICE, M.A. et al. Different measures of energetic efficiency and their phenotypic relationships with growth feed intake and ultrasound and carcass merit in hybrid cattle. J. Anim. Sci., v.82, p.2451-2459, 2004.

NUTRIENT requirements of beef cattle. Washington: National Academies Press, 2016.
ROBINSON, D.L.; ODDY, V.H. Genetics parameters for feed efficiency, fatness, muscle area, and feeding behavior of feedlot finishinged beef cattle. Livest. Prod. Sci., v.90, p.255-270, 2004.

SANTANA, M.H.A.; ROSSI JUNIOR, P.; ALMEIDA, R. et al. Feed efficiency and its correlations with carcass traits measured by ultrasound in Nellore bulls. Livest. Sci., v.145, p.252-257, 2012.

SANTANA, M.H.A; GOMES, R.C.; FERRAZ, J.B.S. et al. Medidas de eficiência alimentar para avaliação de bovinos de corte. Sci. Agrar. Paranaensis, v.13, p.95-107, 2014.

SAS onlineDOC® 9.1.3, Cary: SAS Inst., 2004.

ZAR, J.H. Biostatiscal analysis. New Jersey: Prentice-Hall, 1999. 960p. 